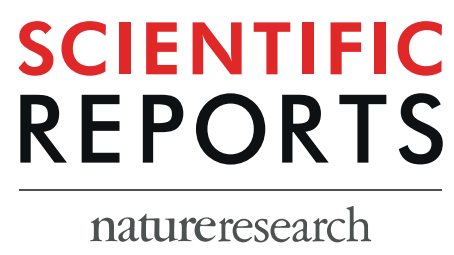

Received: 3 July 2019

Accepted: 11 September 2019

Published online: 24 September 2019

\section{Diagnostic and Prognostic Value of Soluble Urokinase-type Plasminogen Activator Receptor (suPAR) in Focal Segmental Glomerulosclerosis and Impact of
Detection Method}

Wolfgang Winnicki ${ }^{1}{ }^{1}$, Gere Sunder-Plassmann ${ }^{1}$, Gürkan Sengölge ${ }^{1}$, Ammon Handisurya ${ }^{1}$, Harald Herkner ${ }^{2}$, Christoph Kornauth ${ }^{3}$, Bernhard Bielesz $\mathbb{D}^{1}$, Ludwig Wagner ${ }^{1}$, Željko Kikić ${ }^{1}$, Sahra Pajenda ${ }^{1}$, Thomas Reiter $\mathbb{1}^{1}{ }^{1}$, Benjamin Schairer ${ }^{1}$ \& Alice Schmidt ${ }^{1}$

The plasma soluble urokinase-type plasminogen activator receptor (sUPAR) is a biomarker for focal segmental glomerulosclerosis (FSGS), but its value is under discussion because of ambiguous results arising from different ELISA methods in previous studies. The aim of this study was to compare diagnostic performance of two leading suPAR ELISA kits and examine four objectives in 146 subjects: (1) plasma sUPAR levels according to glomerular disease (primary, secondary and recurrent FSGS after kidney transplantation, other glomerulonephritis) and in healthy controls; (2) suPAR levels based on glomerular filtration rate; (3) sensitivity and specificity of suPAR for FSGS diagnosis and determination of optimal cut-offs; (4) suPAR as prognostic tool. Patients with FSGS showed significant higher sUPAR values than patients with other glomerulonephritis and healthy individuals. This applied to subjects with and without chronic kidney disease. Although both suPARnostic TM assay and Quantikine Human uPAR ELISA Kit exerted high sensitivity and specificity for FSGS diagnosis, their cut-off values of $4.644 \mathrm{ng} / \mathrm{mL}$ and $2.789 \mathrm{ng} / \mathrm{mL}$ were significantly different. Higher suPAR was furthermore predictive for progression to end-stage renal disease. In summary, suPAR values must be interpreted in the context of population and test methods used. Knowing test specific cut-offs makes SUPAR a valuable biomarker for FSGS.

Focal segmental glomerulosclerosis (FSGS) represents up to 20 percent of glomerular disease and is a leading cause of end-stage renal failure ${ }^{1}$. It affects native kidneys as well as renal allografts and the rate of recurrence of disease after transplantation is close to $30 \%$ in FSGS patients ${ }^{2}$.

The pathophysiology and etiology of FSGS is still unclear ${ }^{3}$. However, it has been speculated that certain circulating factors might contribute to the initiation of renal injury ${ }^{4}$ as evidenced by recurrence of FSGS after kidney transplantation $^{5}$ and the induction of remission after plasma exchange in subjects with recurrent FSGS early post renal transplantation ${ }^{6}$. Further evidence of potential circulating factors is the induction of proteinuria in kidneys of rats by administration of serum from FSGS patients ${ }^{7}$ and the transmission of FSGS from mother to child ${ }^{8}$. In addition, it was reported that re-transplantation of a renal allograft with rapidly recurrent FSGS in a recipient with primary FSGS as underlying disease into a second recipient without native FSGS resulted in complete remission ${ }^{9,10}$.

Several proteins have been suggested as the causative circulating factors responsible for the occurrence of primary FSGS or recurrence of disease after kidney transplantation, including cardiotrophin-like cytokine-1

\footnotetext{
${ }^{1}$ Department of Medicine III, Division of Nephrology and Dialysis, Medical University of Vienna, Vienna, Austria. ${ }^{2}$ Department of Emergency Medicine, Medical University of Vienna, Vienna, Austria. ${ }^{3}$ Clinical Institute of Pathology, Medical University of Vienna, Vienna, Austria. Correspondence and requests for materials should be addressed to B.B. (email: bernhard.bielesz@meduniwien.ac.at)
} 
$(\mathrm{CLC}-1)^{11}$, vasodilator-stimulated phosphoprotein $(\mathrm{VASP})^{12}$, anti-CD40 antibodies ${ }^{13}$ and soluble urokinase-type plasminogen activator receptor (suPAR) ${ }^{14}$. The most investigated factor in this context to date is suPAR ${ }^{15}$.

The soluble urokinase-type plasminogen activator receptor (suPAR) is derived from the urokinase-type plasminogen activator receptor ( $\mathrm{UPAR}$ ), a membrane bound receptor expressed at the surface of multiple cell types such as immune cells and vascular endothelial cells ${ }^{16}$. By cleavage of its glyxosylphospatidylinositol anchor, uPAR can be released from the plasma membrane as soluble multi-domain signaling molecule (suPAR $)^{5}$. In proteinuric kidney diseases, in particular FSGS, uPAR expression in the kidney and suPAR concentrations in plasma are elevated. As a result of high suPAR and consequential podocyte $\beta 3$ integrin activation, it effects podocyte foot process effacement and disruption of the glomerular barrier leading to proteinuria ${ }^{16}$.

The suPAR levels can be detected in plasma, serum, urine and other body fluids ${ }^{17}$ and are stable in healthy individuals. However, increased plasma levels can be detected not only in FSGS patients, but also under various disease conditions, such as sepsis ${ }^{18}$, liver cirrhosis ${ }^{19}$, immunological disorders ${ }^{20}$ and malignancies ${ }^{21}$ suggesting a role of suPAR as acute phase protein ${ }^{17}$.

The impact of circulating suPAR as causative factor for FSGS is yet under debate. In initial studies, using a proposed suPAR cut-off level of $3.0 \mathrm{ng} / \mathrm{mL}$, increased suPAR levels were identified in up to two-thirds of patients with FSGS ${ }^{22}$, which was significantly higher compared to patients with other forms of glomerular diseases ${ }^{14}$. Furthermore, high suPAR levels were reported to be predictive for recurrence of FSGS after kidney transplantation $^{14}$. It has therefore been suggested that suPAR may represent the circulating factor causing FSGS ${ }^{22}$ and, as a biomarker, may support the often sophisticated FSGS diagnosis.

The diagnosis of FSGS is based on histopathologic findings in representative kidney biopsies and accordingly analysis of at least 15 serial sections is recommended ${ }^{23}$. The quality of a renal biopsy depends on the number of glomeruli and 10-15 glomeruli are considered optimal ${ }^{24}$. The injury of podocytes initiates the disease process of FSGS, leading to the classical focal distribution of sclerosis with a segmental pattern within the glomeruli ${ }^{25}$. However, due to the focal and segmental characteristics of FSGS, histopathological features may be missed and depend on biopsy site and time of biopsy. Biopsy results do not always reflect clinical manifestations ${ }^{3,26}$, therefore, additional tests may facilitate diagnosis of FSGS ${ }^{3}$.

The usefulness of suPAR as a biomarker is promising, but still a matter of debate due to ambiguous results in previous studies which have shown that suPAR levels depended on the degree of renal insufficiency, did not correlate with the extent of proteinuria and were also increased in other kidney diseases than FSGS ${ }^{27-30}$. Furthermore, the role of suPAR in progression of FSGS and chronic kidney disease is discussed in the scientific community ${ }^{1,31,32}$. Varying results in former investigations might partly arise from application of different ELISA kits using differing reagents and antibodies. The aim of our study was therefore to compare the diagnostic and prognostic performance of two leading suPAR ELISA kits that use different capture and detection antibodies of non-identical hosts. We have determined four outcomes using both Elisa kits side-by-side and analyzed their results:

(1) plasma suPAR levels according to glomerular disease (primary FSGS, secondary FSGS, recurrence of FSGS after kidney transplantation, other glomerulonephritis (GN) and in healthy controls; (2) plasma suPAR levels according to kidney function (eGFR); (3) sensitivity and specificity of suPAR levels for FSGS diagnosis after determination of an optimal cut-off value and (4) assessment of suPAR as prognostic marker.

\section{Methods}

Study design. This retrospective cross-sectional study was conducted to compare plasma levels of suPAR in patients with FSGS, other forms of glomerulonephritis and healthy individuals. We further compared suPAR levels in patients with primary FSGS, secondary FSGS and recurrence of FSGS after kidney transplantation.

Study population and recruitment. We measured plasma suPAR in 146 adults over 18 years. Overall 27 patients with primary FSGS, 21 subjects with secondary FSGS and 6 patients with recurrence of FSGS after renal transplantation with clinical and histologic data recorded at the Medical University of Vienna were included in this study. The primary sample collection was carried out between 06/2013 and 04/2015 and follow-ups were performed until 12/2018. Clinical and histological data were obtained at the date of presentation. Moreover, 60 patients with other forms of GN and 32 healthy individuals were used as age- and gender-matched controls (Table 1). Patients with "Other GN" included subjects with membranous GN, minimal change GN, immune complex GN, anti-GBM GN, IgA GN, Lupus GN, interstitial GN and patients with unspecified GN as given in Supplementary Table S1.

Exclusion criteria were age $<18$ years, active inflammatory disease, chronic liver disease, malignancy, pregnancy and any illicit drug use. Patients with FSGS or other GN whose renal biopsy specimen contained less than 10 glomeruli were also excluded.

We first examined the association between baseline suPAR levels and measures of kidney function (eGFR, urine protein/creatinine ratio and albumin/creatinine ratio) in all study subjects. We also investigated the association of suPAR and change in eGFR and further clinical parameters and outcomes during follow-up in all 54 FSGS patients during a follow-up period of 5 years.

Histopathology. The kidney biopsy was performed at the time of diagnosis. Biopsy specimens were formalin-fixed, paraffin embedded, cut at $2 \mu \mathrm{m}$ and histologically evaluated by light microscopy, indirect immunohistochemistry and electron microscopy following institutional guidelines. All of the biopsy slides were reviewed by two pathologists blinded to clinical data of the patients.

Sample collection. Fasting venous blood samples were collected of 54 FSGS patients and samples from 92 age- and gender matched patients with other forms of GN or healthy individuals as normal controls. Plasma 


\begin{tabular}{|c|c|c|c|c|c|c|c|}
\hline & FSGS total & Primary FSGS & $\begin{array}{l}\text { Secondary } \\
\text { FSGS }\end{array}$ & $\begin{array}{l}\text { Recurrence of } \\
\text { FSGS in RTX }\end{array}$ & Other GN & $\begin{array}{l}\text { Healthy } \\
\text { controls }\end{array}$ & P-value ${ }^{\circ}$ \\
\hline Number of subjects & 54 & 27 & 21 & 6 & 60 & 32 & \\
\hline \multicolumn{8}{|l|}{ Characteristics } \\
\hline - Age (years) & $45.1 \pm 14.7$ & $48.4 \pm 13.0$ & $45.1 \pm 15.4$ & $30.2 \pm 11.1$ & $47.4 \pm 16.6$ & $42.3 \pm 8.3$ & 0.44 \\
\hline - Gender - male number (\%) & $33(61)$ & $16(59)$ & $15(71)$ & $2(33)$ & $32(53)$ & $16(50)$ & 0.57 \\
\hline - White - number (\%) & $48(89)$ & $25(93)$ & $17(81)$ & $6(100)$ & $57(95)$ & $30(94)$ & 0.19 \\
\hline - MAP (mmHG) & $97 \pm 9$ & $98 \pm 6$ & $103 \pm 6$ & $88 \pm 16$ & $90 \pm 11$ & $92 \pm 6$ & 0.14 \\
\hline - Treated with RAS blockade - number (\%) & $44(81)$ & $22(81)$ & $17(81)$ & $5(83)$ & $43(72)$ & $0(0)$ & 0.42 \\
\hline $\begin{array}{l}\text { - Treated with immunosuppression - } \\
\text { number (\%) }\end{array}$ & $34(63)$ & $21(78)$ & $7(33)$ & $6(100)$ & $29(48)$ & $0(0)$ & 0.06 \\
\hline \multicolumn{8}{|l|}{ Coexisting or prior illness - number (\%) } \\
\hline - Arterial hypertension - number (\%) & $48(89)$ & $24(89)$ & $18(85)$ & $6(100)$ & $48(80)$ & $0(0)$ & 0.35 \\
\hline - Diabetes mellitus - number (\%) & $12(22)$ & $6(22)$ & $5(24)$ & $1(17)$ & $6(10)$ & $0(0)$ & 0.16 \\
\hline \multicolumn{8}{|l|}{ Laboratory parameters } \\
\hline - Serum albumin $(\mathrm{g} / \mathrm{dL})$ & $37.47 \pm 7.27$ & $35.81 \pm 8.52$ & $40.39 \pm 3.69$ & $35.23 \pm 8.13$ & $36.47 \pm 7.61$ & ns & 0.50 \\
\hline - Serum total cholesterol (mg/dL) & $218.40 \pm 62.08$ & $230.24 \pm 70.39$ & $208.63 \pm 55.89$ & $200.00 \pm 35.60$ & $234.65 \pm 67.46$ & ns & 0.28 \\
\hline - Serum C-reactive protein (mg/dL) & $0.41 \pm 0.47$ & $0.38 \pm 0.56$ & $0.48 \pm 0.41$ & $0.27 \pm 0.20$ & $0.23 \pm 0.25$ & $0.09 \pm 0.08$ & 0.02 \\
\hline - Serum creatinine (mg/dL) & $2.19 \pm 1.89$ & $1.80 \pm 1.22$ & $2.31 \pm 2.01$ & $3.51 \pm 3.30$ & $1.40 \pm 1.35$ & $0.86 \pm 0.11$ & 0.0004 \\
\hline - eGFR $\left(\mathrm{mL} / \mathrm{min} / 1.73 \mathrm{~m}^{2}\right)$ & $52.37 \pm 31.61$ & $55.90 \pm 32.13$ & $51.61 \pm 32.04$ & $39.12 \pm 28.87$ & $75.85 \pm 33.48$ & $99.95 \pm 15.89$ & 0.0003 \\
\hline - Microscopic hematuria, n (\%) & $8(15)$ & $3(11)$ & $3(14)$ & $2(33)$ & $12(20)$ & ns & 0.47 \\
\hline - Urine albumin/creatinine ratio $(\mathrm{mg} / \mathrm{g})$ & $1582 \pm 1728$ & $1627 \pm 1774$ & $1337 \pm 1531$ & $2376 \pm 2272$ & $1813 \pm 2137$ & ns & 0.89 \\
\hline - Urine protein/creatinine ratio (mg/g) & $2316 \pm 2325$ & $2435 \pm 2331$ & $1848 \pm 2101$ & $3422 \pm 2986$ & $2526 \pm 2913$ & ns & 0.85 \\
\hline \multicolumn{8}{|l|}{ Plasma suPAR levels (ng/mL) } \\
\hline SuPAR (suPARnostic ${ }^{\mathrm{TM}}$ assay)* & $6.58 \pm 2.25$ & $6.65 \pm 2.26$ & $6.40 \pm 2.46$ & $6.96 \pm 1.61$ & $3.28 \pm 1.47$ & $1.53 \pm 0.60$ & 0.0001 \\
\hline SuPAR (Quantikine Human uPAR)** & $3.54 \pm 1.03$ & $3.60 \pm 1.14$ & $3.44 \pm 0.85$ & $3.65 \pm 1.26$ & $3.07 \pm 1.06$ & $2.07 \pm 0.50$ & 0.0026 \\
\hline
\end{tabular}

Table 1. Baseline Characteristics and plasma suPAR levels of Study Patients. Plus-minus values are means \pm standard deviation. Numbers in brackets indicate percentage. Abbreviations: eGFR, estimated glomerular filtration rate; FSGS, focal segmental glomerulosclerosis; GN, glomerulonephritis; MAP, mean arterial pressure; ns, not studied; RAS, renin angiotensin system; RTX, renal transplantation. ${ }^{\circ} \mathrm{P}$-value of FSGS total vs. Other GN. *Plasma suPAR value $(\mathrm{ng} / \mathrm{mL})$ measured by suPARnostic ${ }^{\mathrm{TM}}$ assay. $* *$ Plasma suPAR value (ng/mL) measured by Quantikine Human uPAR ELISA Kit.

was separated within 30 minutes following blood draw and stored in multiple aliquots at $-80^{\circ} \mathrm{C}$ until analyzed. Repeated freezing and thawing cycles of the samples were avoided.

Plasma suPAR measurement. Concentration of plasma suPAR in human subjects were detected using the suPARnostic ${ }^{\mathrm{TM}}$ assay (Virogates, Copenhagen, Denmark) and the Quantikine Human uPAR Immunoassay (R\&D Systems, Minneapolis, MN, USA) ${ }^{1}$, following the manufacturers protocol. Both kits are measuring suPAR concentrations and minimum detectable levels are $100 \mathrm{pg} / \mathrm{mL}$ and $33 \mathrm{pg} / \mathrm{mL}$, respectively. A schematic illustration of both kits is shown as Supplementary Fig. S1.

suPARnostic ${ }^{\mathrm{TM}}$ assay (Virogates, Copenhagen, Denmark). The suPARnostic kit (ViroGates A/S, Birkerød, Denmark) represents a double monoclonal antibody sandwich assay that measures total suPAR, including both full-length and cleaved forms. Plates are coated with catching rat monoclonal antibody. Horseradish peroxidase (HRP)-labeled detection mouse monoclonal antibody is mixed in a volume of $225 \mu \mathrm{L}$ of Peroxidase Conjugate solution together with $25 \mu \mathrm{L}$ of plasma. Out of this, $100 \mu \mathrm{L}$ was transferred (in duplicates) to the pre-coated immunoassay plate and incubated for one hour. After washing procedures, $100 \mu \mathrm{L}$ of the TMB substrate $\left(3,3^{\prime}, 5,5^{\prime}\right.$ tetramethylbenzidine) was added to each well. The substrate/chromogen reaction was stopped after 10 minutes by the Stop Solution $\left(0.45 \mathrm{M}\right.$ sulphuric acid $-\mathrm{H}_{2} \mathrm{SO}_{4}$ ) All incubations were performed at room temperature in the dark.

Plasma sample concentrations were calculate according to the standard curve included in each plate. The intra-assay variation of the kit is given as $2.8 \%$ and the inter-assay variation as $9.2 \%{ }^{33}$. Samples were randomly distributed between kits of five different lot numbers and were all measured in duplicates. All samples had duplicate coefficients of variants $(\mathrm{CVs})<10 \%$.

Quantikine Human uPAR Immunoassay (R\&D Systems, Minneapolis, MN, USA). With regard to the Quantikine Human uPAR Immunoassay, $100 \mu \mathrm{L}$ assay diluent was applied to every well of 96-well polystyrene microplates that were precoated with a mouse monoclonal antibody, followed by $50 \mu \mathrm{L}$ plasma and incubated for 2 hours at ambient temperature. After incubation and washing procedure, horseradish peroxidase-conjugated polyclonal antibodies against uPAR were admixed and incubated for 2 hours at ambient temperature. After repeated washing, a substrate solution was applied to every well and incubated for further 30 minutes at ambient temperature 
while protecting it from light. Finally, $50 \mu \mathrm{L}$ of stop solution was admixed in every well and the absorbance was detected at $450 \mathrm{~nm}$ over 30 minutes using an ELISA reader ${ }^{1,34}$. The intra-assay variation of the kit is given as $7.5 \%$ and the inter-assay variation as $5.9 \%$. Samples were randomly distributed between kits of five different lot numbers and were all measured in duplicates. All samples had duplicate coefficients of variants $(\mathrm{CVs})<10 \%$.

Study procedures. Laboratory diagnostic for routine parameters. All laboratory parameters were determined at the Department of Laboratory Medicine, Medical University of Vienna, which has a certified (ISO 9001:2008) and accredited (ISO 15189:2008) quality management system. The eGFR was calculated using the chronic kidney disease EPI equation ${ }^{35}$.

Outcomes. The current study investigated the association of FSGS status and suPAR levels in plasma measured by different ELISA kits. Furthermore, a prognostic assessment of suPAR was performed for eGFR dynamics, ESRD and death.

Statistical analyses. Categorized data are presented as absolute counts and relative frequencies (percent). We presented continuous data as means \pm standard deviation (SD). To compare baseline variables between study groups we used the Mann-Whitney U test, the median test or the Fisher's exact test, as appropriate. To compare difference in suPAR levels from different test kits we used the $\mathrm{t}$-test with $\mathrm{H}_{0}$ : delta $=0$, calculating disease group specific estimates with respective $95 \%$ confidence intervals.

We calculated standard metrics of diagnostic test accuracy including sensitivity and specificity of suPAR levels (index tests) for identifying patients with FSGS (reference standard) independently. We estimated the optimal cut-off by maximizing the sensitivity-specificity product according to Liu's method ${ }^{36}$ using the adjustment method suggested by Fluss et al. ${ }^{37}$. The confidence interval for the cut-off value was estimated by bootstrapping.

For prognostic assessment of suPAR (main co-variable) for eGFR dynamics, ESRD, and death (individual outcomes) multivariable regression analyses were employed. For eGFR dynamics linear regression, for ESRD and death logistic regression were used. Co-variables were sex (male vs female), age (years), eGFR ( $\mathrm{mL} / \mathrm{min}$ ) and initial urine protein/creatinine ratio $(\mathrm{mg} / \mathrm{g})$. We report the estimates from the regression analysis with a $95 \%$ confidence interval and the corresponding P-values from the Wald test. We furthermore conducted a sensitivity analysis in which differences in eGFR, urine protein/creatinine ratio and albumin/creatinine ratio values were set to zero after loss of renal function.

Statistical analysis and data management was carried out with Stata 14 for Mac (Stata Corp, College Station, TX, USA), GraphPad Prism (ㄷ GraphPad Prism version 7.00 for Windows, GraphPad Software, La Jolla California, USA) and MS Excel (@ Microsoft, Redmont, WA).

All tests conducted were two-sided and a P-value below 0.05 was considered significant.

\section{Results}

Study population. This study analyzed demographic data, laboratory parameters and plasma suPAR levels of 54 patients with FSGS (27 patients with primary FSGS, 21 with secondary FSGS and 6 with recurrence of FSGS in the renal allograft) and 92 controls (60 patients with glomerulonephritis other than FSGS termed "Other GN" and 32 healthy individuals) as measured by two leading ELISA assays. Demographic and clinical data of the entire study cohort are given in Table 1 .

Plasma suPAR level according to glomerular disease. Patients with FSGS presented in general higher suPAR levels $[\mathrm{ng} / \mathrm{mL}]$ compared to other GN patients and healthy individuals $(6.58 \pm 2.25$, vs. $3.28 \pm 1.47$ vs. $1.53 \pm 0.60 \mathrm{ng} / \mathrm{mL} ; \mathrm{p}=0.0001$ ) as measured with the suPARnostic ${ }^{\mathrm{TM}}$ assay (Table 1, Fig. 1a). Also when using the Quantikine Human uPAR ELISA Kit significantly higher suPAR levels [ng/mL] were obtained for FSGS patients compared to patients with other GN and healthy volunteers $(3.54 \pm 1.03$, vs. $3.07 \pm 1.06 \mathrm{vs} .2 .07 \pm 0.50 \mathrm{ng} / \mathrm{mL}$; $\mathrm{p}=0.0026$ ), (Table 1, Fig. 1b). No difference was found between plasma suPAR levels of patients with primary FSGS, secondary FSGS or recurrence of FSGS post transplantation. A comprehensive subgrouping of patients with FSGS, other GN and healthy individuals is depicted in Supplementary Table S1.

In a group-specific comparison of the suPARnostic ${ }^{\mathrm{TM}}$ assay and the Quantikine Human uPAR-Immunoassay the mean difference of suPAR levels in FSGS patients was $3.04 \pm 1.5 \mathrm{ng} / \mathrm{mL}(44 \%$; $95 \% \mathrm{CI}, 40$ to $48 ; \mathrm{p}<0.001)$, in patients with other GN $0.21 \pm 1.1 \mathrm{ng} / \mathrm{mL}(-2 \%$; $95 \% \mathrm{CI},-11$ to $6 ; \mathrm{p}<0.62)$ and in healthy controls $-0.54 \pm 0.3 \mathrm{ng} / \mathrm{mL}(-43 \% ; 95 \% \mathrm{CI},-54$ to $-32 ; \mathrm{p}<0.001)$.

Plasma suPAR level depending on kidney function. The mean eGFR was significantly lower in FSGS patients compared to patients with other GN or healthy volunteers, which might partially account for the difference of suPAR levels between groups. Multiple regression analysis showed that baseline suPAR level was significantly correlated with eGFR $(\mathrm{p}=0.001)$ which remained significant after multivariable adjustment as shown in Table 2 for both ELISA tests. No significant association was observed between plasma suPAR and gender, age, proteinuria, presence of microscopic hematuria, history of renal transplantation or duration of FSGS disease.

To test the validity of suPAR levels in patients without chronic kidney disease, we excluded 54 patients with eGFR below $60 \mathrm{~mL} / \mathrm{min}$, in accordance with the definition of chronic kidney disease, for a subanalysis. In the remaining 92 study subjects with eGFR $\geq 60 \mathrm{~mL} / \mathrm{min}$ a significant difference in suPAR levels [ng/mL] between FSGS and other GN patients $\left(5.05 \pm 1.35\right.$ vs. $2.91 \pm 0.94 \mathrm{ng} / \mathrm{mL}, \mathrm{p}=0.001$ for the suPARnostic ${ }^{\mathrm{TM}}$ assay and $2.91 \pm 0.48$ vs. $2.61 \pm 0.49 \mathrm{ng} / \mathrm{mL}, \mathrm{p}=0.036$ for the Quantikine Human uPAR ELISA Kit) was confirmed. This also applied to the comparison between FSGS patients and healthy controls $(5.05 \pm 1.35 \mathrm{vs} .1 .53 \pm 0.60 \mathrm{ng} / \mathrm{mL}$, $\mathrm{p}=0.001$ for the suPARnostic ${ }^{\mathrm{TM}}$ assay and $2.91 \pm 0.48 \mathrm{vs} .2 .07 \pm 0.50 \mathrm{ng} / \mathrm{mL}, \mathrm{p}=0.001$ for the Quantikine Human 

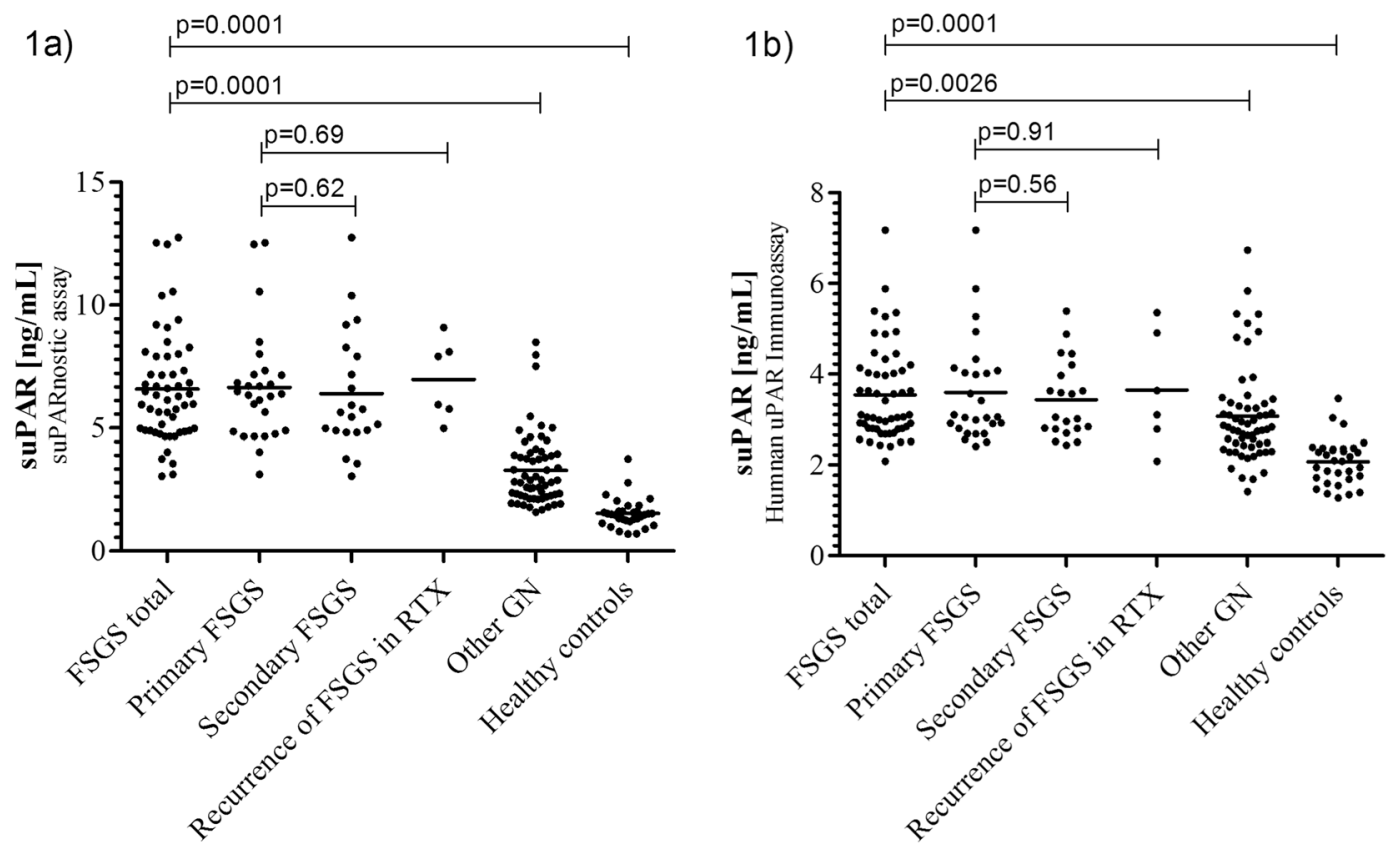

Figure 1. Plasma suPAR levels in patients with FSGS total, primary FSGS, secondary FSGS, recurrence of FSGS after renal transplantation, other glomerulonephritis and healthy controls. (a) Results from suPARnostic ${ }^{\mathrm{TM}}$ assay: plasma suPAR levels of patients with FSGS (FSGS total, $6.58 \pm 2.25 \mathrm{ng} / \mathrm{mL}$ ) were significantly higher than in patients with other GN $(3.28 \pm 1.47 \mathrm{ng} / \mathrm{mL})$ and healthy controls $(1.53 \pm 0.60 \mathrm{ng} / \mathrm{mL})$. (b) Results from Quantikine Human uPAR ELISA Kit: suPAR levels in patients with FSGS (FSGS total, $3.54 \pm 1.03 \mathrm{ng} /$ $\mathrm{mL})$ were significantly higher than in patients with other $\mathrm{GN}(3.07 \pm 1.06 \mathrm{ng} / \mathrm{mL})$ and healthy controls $(2.07 \pm 0.50 \mathrm{ng} / \mathrm{mL})$. There was no significant difference in plasma suPAR levels between patients with primary FSGS, secondary FSGS and recurrence of FSGS after renal transplantation. P-values are based on the MannWhitney U test. Abbreviations: FSGS, focal segmental glomerulosclerosis; GN, glomerulonephritis; RTX, renal transplantation.

UPAR ELISA Kit). Of note, renal function parameters did not differ between FSGS, other GN patients and healthy controls in this subanalysis (eGFR $90.2 \pm 19.1$. vs. $93.5 \pm 20.0$ vs. $99.9 \pm 15.9 \mathrm{~mL} / \mathrm{min}$; FSGS vs. other GN: $\mathrm{p}=0.60$, FSGS vs. healthy controls: $\mathrm{p}=0.12$; Table 3 ). Characteristics and laboratory parameters of FSGS patients with eGFR above $60 \mathrm{~mL} / \mathrm{min}$ and below are presented in Supplementary Table S2.

Sensitivity and specificity of plasma suPAR for FSGS diagnosis. We performed a receiver operating characteristic (ROC) analysis to determine whether suPAR is a potent marker to diagnose and differentiate FSGS.

By identifying the point at which the difference between sensitivity and 1-specificity is maximal, we determined that the optimal cut-off value is $4.644 \mathrm{ng} / \mathrm{mL}$ of the suPAR concentration measured by the suPARnostic ${ }^{\mathrm{TM}}$ assay for FSGS vs. non-FSGS patients [95\% CI 4.02 to 5.27]. For this cut-off value, the sensitivity and specificity of the test were both 0.91 with an AUC of 0.946 [95\% CI 0.91 to 0.98] (Fig. 2a).

Using the Quantikine Human uPAR ELISA Kit the optimal cut-off is $2.789 \mathrm{ng} / \mathrm{mL}$ for FSGS vs. non-FSGS patients [95\% CI 2.35 to 3.22]. For this cut-off value, the sensitivity and specificity of the test were 0.80 and 0.64 , respectively with an AUC of 0.763 [95\% CI 0.69 to 0.83] (Fig. 2b).

These results suggest that both kits measuring suPAR can be used to distinguish FSGS from non-FSGS, with the suPARnostic ${ }^{\mathrm{TM}}$ assay performing a higher differentiation in our analysis.

An illustration of suPAR cut-off values and possible false negative test results in patients with FSGS depending on the test method used is shown in Supplementary Fig. S2.

The ROC analysis of a subanalysis of subjects with eGFR $\geq 60 \mathrm{~mL} / \mathrm{min}$ is presented in Supplementary Fig. S3.

Prognostic assessment of plasma suPAR levels. A multivariate analysis was developed including age, sex, eGFR and urine protein/creatinine ratio to test the prognostic value of plasma suPAR in FSGS patients. With each suPAR increase by one point $[\mathrm{ng} / \mathrm{mL}]$, measured with the suPARnostic ${ }^{\mathrm{TM}}$ assay following changes were detected: decrease in the mean annual delta eGFR by $0.098 \mathrm{~mL} / \mathrm{min}(95 \% \mathrm{CI}-0.66$ to 0.46$), \mathrm{p}=0.729$; an increase in the mean annual delta urine protein/creatinine ratio by $48.3 \mathrm{mg} / \mathrm{g}(95 \% \mathrm{CI}-44.5$ to 141$), \mathrm{p}=0.30$ as well as in the mean annual delta urine albumin/creatinine ratio by $9.2 \mathrm{mg} / \mathrm{g}(95 \% \mathrm{CI}-93.5$ to 111.9$), \mathrm{p}=0.86$. These results were not significant. During the 5-year follow-up, 12 out of 54 patients with FSGS developed ESRD and 4 FSGS patients died. With each suPAR increase by one point $[\mathrm{ng} / \mathrm{mL}]$ the odds ratio for death increased by $1.15 \pm 0.24$ ( $95 \%$ CI 0.76 to 1.72 ), $\mathrm{p}=0.52$. A significant association could be shown between suPAR and progression to ESRD, as with each suPAR increase by one point $[\mathrm{ng} / \mathrm{mL}]$ the odds ratio for ESRD increased by $1.34 \pm 0.19(95 \%$ 


\begin{tabular}{|c|c|c|c|c|}
\hline Variable & $\begin{array}{l}\text { Regression coefficient }(95 \% \mathrm{CI}) \text { with } \\
\text { suPAR adjusted }{ }^{\mathrm{a}} \text { suPARnostic }{ }^{\mathrm{MM}} \text { assay* }\end{array}$ & P-value & \begin{tabular}{|l|} 
Regression coefficient $(95 \%$ CI) with suPAR \\
adjusted $^{\mathrm{a}}$ Quantikine Human uPAR ELISA Kit $* *$
\end{tabular} & P-value \\
\hline Gender (male) & $-0.598(-1.748$ to 0.553$)$ & 0.302 & $-0.341(-0.876$ to 0.193$)$ & 0.205 \\
\hline Age (years) & $0.009(-0.028$ to 0.046$)$ & 0.641 & $0.015(-0.002$ to 0.032$)$ & 0.089 \\
\hline eGFR $\left(\mathrm{mL} / \mathrm{min} / 1.73 \mathrm{~m}^{2}\right)$ & $-0.043(-0.061$ to -0.025$)$ & 0.001 & $-0.018(-0.026$ to -0.010$)$ & 0.001 \\
\hline Serum creatinine $(\mathrm{mg} / \mathrm{dL})$ & $0.074(-0.576$ to 0.428$)$ & 0.769 & $-0.001(-0.234$ to 0.233$)$ & 0.996 \\
\hline Serum albumin $(\mathrm{g} / \mathrm{dL})$ & $0.003(-0.099$ to 0.105$)$ & 0.952 & $-0.002(-0.049$ to 0.046$)$ & 0.941 \\
\hline Serum C-reactive protein $(\mathrm{mg} / \mathrm{dL})$ & $0.492(-0.661$ to 1.645$)$ & 0.395 & $0.101(-0.438$ to 0.640$)$ & 0.709 \\
\hline Serum total cholesterol $(\mathrm{mg} / \mathrm{dL})$ & $-0.004(-0.014$ to 0.006$)$ & 0.435 & $-0.001(-0.005$ to 0.004$)$ & 0.757 \\
\hline Urine protein/creatinine ratio $(\mathrm{mg} / \mathrm{g})$ & $0.001(-0.001$ to 0.001$)$ & 0.142 & $0.001(-0.001$ to 0.001$)$ & 0.100 \\
\hline Urine albumin/creatinine ratio $(\mathrm{mg} / \mathrm{g})$ & $0.001(-0.001$ to 0.002$)$ & 0.871 & $-0.001(-0.001$ to 0.002$)$ & 0.942 \\
\hline Presence of microscopic hematuria & $-0.466(-2.222$ to 1.290$)$ & 0.595 & $-0.133(-0.927$ to 0.661$)$ & 0.738 \\
\hline Presence of hypertension & $-1.843(-4.227$ to 0.541$)$ & 0.125 & $-0.026(-1.130$ to 1.077$)$ & 0.961 \\
\hline Presence of diabetes & $0.855(-0.726$ to 2.437$)$ & 0.280 & $0.476(-0.200$ to 1.151$)$ & 0.162 \\
\hline History of renal transplantation & $0.592(-0.611$ to 1.795$)$ & 0.327 & $0.111(-0.453$ to 0.674$)$ & 0.695 \\
\hline Duration of disease (months) & $0.023(-0.099$ to 0.146$)$ & 0.702 & $0.026(-0.031$ to 0.082$)$ & 0.367 \\
\hline
\end{tabular}

Table 2. Association of baseline variables with plasma suPAR levels, measured by two independent ELISA tests,

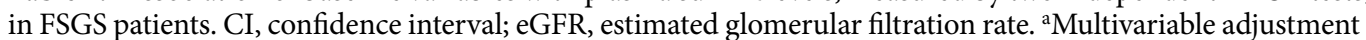
for sex (male/female), age (years), eGFR and urine protein/creatinine ratio. *Plasma suPAR values were measured by suPARnostic ${ }^{\mathrm{TM}}$ assay. **Plasma suPAR values were measured by Quantikine Human uPAR ELISA Kit.

\begin{tabular}{|l|l|l|l|l|l|}
\hline & FSGS total & Other GN & $\begin{array}{l}\text { Healthy } \\
\text { controls }\end{array}$ & $\begin{array}{l}\text { P-value } \\
\text { FSGS vs. } \\
\text { Other GN }\end{array}$ & $\begin{array}{l}\text { P-value } \\
\text { FSGS vs. } \\
\text { Healthy }\end{array}$ \\
\hline Number of subjects & 18 & 42 & 32 & & \\
\hline Age (years) & $45.2 \pm 14.6$ & $46.2 \pm 15.6$ & $42.3 \pm 8.3$ & 0.82 & 0.18 \\
\hline Gender (male/female) & $7 / 11$ & $28 / 15$ & $16 / 16$ & 0.06 & 0.58 \\
\hline Serum creatinine $(\mathrm{mg} / \mathrm{dL})$ & $0.87 \pm 0.18$ & $0.90 \pm 0.19$ & $0.86 \pm 0.11$ & 0.51 & 0.98 \\
\hline eGFR $\left(\mathrm{mL} / \mathrm{min} / 1.73 \mathrm{~m}^{2}\right)$ & $90.2 \pm 19.1$ & $93.5 \pm 20.0$ & $99.9 \pm 15.9$ & 0.60 & 0.12 \\
\hline Plasma suPAR $(\mathrm{ng} / \mathrm{mL}) *$ & $5.05 \pm 1.35$ & $2.91 \pm 0.94$ & $1.53 \pm 0.60$ & 0.001 & 0.001 \\
\hline Plasma suPAR $(\mathrm{ng} / \mathrm{mL}) * *$ & $2.91 \pm 0.48$ & $2.61 \pm 0.49$ & $2.07 \pm 0.50$ & 0.036 & 0.001 \\
\hline
\end{tabular}

Table 3. Plasma suPAR levels in patients with FSGS, other GN and healthy controls with glomerular filtration rate $\geq 60 \mathrm{~mL} / \mathrm{min}$. Abbreviations: FSGS, focal segmental glomerulosclerosis; GN, glomerulonephritis. Plusminus values indicate means \pm standard deviation. *Plasma suPAR value measured by suPARnostic ${ }^{\mathrm{TM}}$ assay. **Plasma suPAR value measured by Quantikine Human uPAR ELISA Kit.

CI 1.01 to 1.77), $\mathrm{p}=0.046$. Corresponding results obtained with the Quantikine Human uPAR ELISA Kit are also given in Supplementary Table S3. A graphical depiction of increasing plasma suPAR levels and progression to ESRD is presented in Supplementary Fig. S4.

Similar results were obtained in a model used as sensitivity analysis in which differences in eGFR, urine albu$\mathrm{min} / \mathrm{creatinine}$ ratio and protein/creatinine ratio values were set to zero after loss of renal function compared to the original model using missing data. (Supplementary Table S3).

\section{Discussion}

This study was conducted to test the diagnostic and prognostic value of suPAR as a biomarker for FSGS. Since conflicting results about the clinical role of suPAR in the literature are derived from different ELISA methods measuring suPAR plasma levels, two of the leading assays were used in a side-by-side comparison and their results were compared in this study.

Our study analyzed plasma suPAR levels in patients with different forms of FSGS (primary and secondary FSGS as well as recurrence after transplantation), other types of glomerulonephritis and healthy subjects. Furthermore, suPAR was assessed according to estimated glomerular filtration rate in patients with and without chronic kidney disease. The prognostic evaluation was performed over a period of five years with respect to renal retention parameters, proteinuria, disease progression and mortality. Our data show that FSGS patients have significantly higher suPAR plasma levels when compared to patients with other forms of GN or healthy controls indicating the significance of suPAR tests in FSGS diagnosis. Furthermore, elevated suPAR values in FSGS patients were found to have a predictive value for disease progression to ESRD. Our results also demonstrate that both commercially available suPARnostic ${ }^{\mathrm{TM}}$ assay and the Quantikine Human uPAR Immunoassay can be used to differentiate FSGS from non-FSGS based on suPAR plasma levels, with the suPARnostic ${ }^{\mathrm{TM}}$ assay being more FSGS specific. Novel test specific cut-offs of $4.644 \mathrm{ng} / \mathrm{mL}$ for the suPARnostic ${ }^{\mathrm{TM}}$ assay and $2.789 \mathrm{ng} / \mathrm{mL}$ for the Quantikine Human uPAR Immunoassay were established for FSGS diagnosis in the entire study population. 

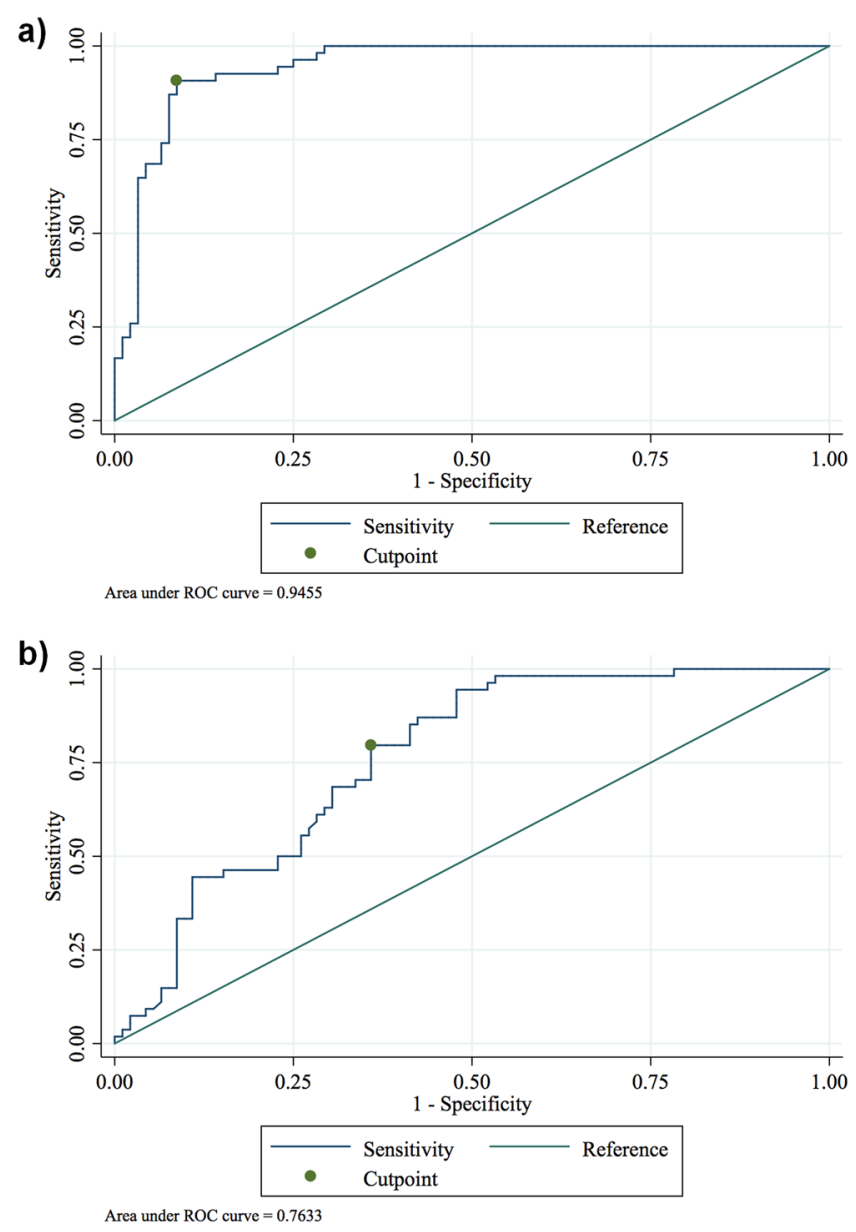

Figure 2. Receiver operating characteristic (ROC) analysis of the plasma soluble urokinase-type plasminogen activator receptor (suPAR) in patients FSGS vs. non-FSGS. (a) For the cut-off value of $4.644 \mathrm{ng} / \mathrm{mL}$ measured by the suPARnostic ${ }^{\mathrm{TM}}$ assay, sensitivity and specificity were both 0.91 with an AUC of 0.946 [95\% CI 0.91 to 0.98]. (b) For the cut-off value of $2.789 \mathrm{ng} / \mathrm{mL}$ measured by the Quantikine Human uPAR ELISA Kit the sensitivity and specificity were 0.80 and 0.64 , respectively with an AUC of 0.763 [95\% CI 0.69 to 0.83].

A number of FSGS studies have used different test methods for the quantitative measurement of suPAR and came to different conclusions. It is noteworthy that fragments of suPAR are not recognized equally by the currently available suPAR ELISA test methods. The separation of uPAR from its GP anchor releases suPAR and further suPAR fragments are generated by additional cleavage between the D1, D2/D3 domains ${ }^{17}$. Furthermore, depending on the amount of glycosylation, the size of the cleaved suPAR fragments ranges from 25 to $50 \mathrm{kDa}^{17}$. This is of central importance: suPAR fragments differ in regard to their biological relevance and are not equally detected in different suPAR assays leading to varying results which are hard to compare among studies ${ }^{5,38,39}$. In our study two ELISA tests were used, the suPARnostic ${ }^{\mathrm{TM}}$ assay and the Quantikine Human uPAR immunoassay. While the suPARnostic ${ }^{\mathrm{TM}}$ assay represents a double monoclonal antibody sandwich assay, the Quantikine Human UPAR immunoassay uses a monoclonal capture antibody and a polyclonal detection antibody. Furthermore, the suPARnostic ${ }^{\text {TM }}$ assay measures both full and cleaved forms of suPAR, which might explain variations in test readings. Thus, although both ELISA kits tested in our study were capable to differentiate FSGS from non-FSGS, test results were not completely identical: in the entire study population of 146 subjects the suPARnostic ${ }^{\mathrm{TM}}$ assay outperfomed the Quantikine Human uPAR-Immunoassay in differentiating FSGS from non-FSGS. This was based on higher suPAR levels in patients with FSGS and lower suPAR levels in healthy volunteers when compared with the Quantikine Human uPAR-Immunoassay. This difference between the two test kits used was attenuated in subjects with eGFR above $60 \mathrm{~mL} / \mathrm{min}$.

Our study demonstrates the importance of suPAR testing in the diagnosis of FSGS. In previous studies, the correlation of suPAR with renal insufficiency was repeatedly discussed critically ${ }^{40}$. This led us to analyze suPAR values in patients with chronic kidney disease (according to the chronic kidney disease (CKD) definition of GFR below $60 \mathrm{~mL} / \mathrm{min}$ ) as well as in patients without CKD. The inverse association of suPAR with eGFR was confirmed in our study; moreover the significance of suPAR in FSGS assessment independent of renal function could be emphasized.

SuPAR may not only be a diagnostic biomarker for FSGS, but also a pathogenic factor for progression of disease $^{26}$. Previously, it has been shown that proteinuria can be reduced by eliminating suPAR from the circulation 
using extracorporeal methods, e.g. plasmapheresis and immunoadsorption. Recently, also hemoadsorption by means of polymeric adsorber columns have been used to remove suPAR ${ }^{38}$. As suPAR has a high affinity to protein A immunoapheresis columns, it is removable from the circulation, at least in part, but also rebounds within days $^{16}$. An established therapy for recurrence of FSGS after transplantation is plasmapheresis or immunoadsorption, in combination with steroids, cyclosporine A and rituximab or alone ${ }^{15,38}$.

Recurrence of FSGS after transplantation manifests in about 30\% of FSGS patients receiving their first kidney transplant ${ }^{2}$ and in up to $80 \%$ of patients after second transplantation with a history of transplant loss due to recurrence of FSGS in the first graft ${ }^{41}$. This can even manifest within hours post transplantation ${ }^{42}$. Since the recurrence of disease is a serious problem, there are attempts to reduce recurrence rates by using induction therapies such as rituximab prior to transplantation in patients with FSGS as underlying disease with high suPAR values ${ }^{43}$. Accordingly, suPAR values in kidney transplant candidates where a high recurrence rate can be assumed could serve as guide to a patient-tailored immunosuppressive therapy, but randomized controlled trials are needed to support this hypothesis.

Furthermore, suPAR measurement could be particularly useful in other clinical situations, where a kidney biopsy for diagnosis is not possible. With the aid of other established biomarkers such as anti-PLA2R antibodies ${ }^{44}$, different forms of GN (membranous nephropathy versus FSGS) can be distinguished, which has in turn therapeutic consequences. Thus, suPAR is of manifold interest in FSGS pathogenesis, diagnosis, therapy and prognosis.

The main limitation of this study is its retrospective design. In addition, our sample size was relatively small due to the monocentric nature of the study. Furthermore, no urine samples were taken that would have allowed further potentially significant analyses ${ }^{34,45}$. Our study was strengthened by a well-characterized cohort of patients with primary FSGS, secondary FSGS, recurrence of FSGS after transplantation, different forms of glomerulonephritis and healthy controls with long follow-up resulting in clear results: plasma suPAR levels do not distinguish between patients with primary and secondary form of FSGS and recurrence of disease after renal transplantation, which is in line with results from previous studies ${ }^{46}$. But suPAR levels are useful to differentiate FSGS patients from patients with other forms of glomerulonephritis and healthy controls, e.g. when renal biopsies are difficult. The independence of suPAR from eGFR has been demonstrated and both the suPARnostic ${ }^{\mathrm{TM}}$ assay and the Quantikine Human uPAR-Immunoassay are capable for FSGS identification. Furthermore, the prognostic value of suPAR for the progression to end-stage renal disease was identified.

The scope of this study was not to establish the role of suPAR in the pathogenesis of FSGS. The main aim was to examine the significance of suPAR in FSGS diagnosis and different courses of disease. Hereby, we describe new cut-offs for suPAR using two different suPAR ELISA assays. The comparison of these two assays, showing relevant differences, may explain varying results obtained in previous studies using different detection assays. Therefore, suPAR values have to be interpreted in the context of population and the test method used. Knowledge of test specific cut-offs makes suPAR a valuable biomarker for FSGS.

\section{Data Availability}

The datasets generated in the context of this paper are made available by the corresponding author on request.

\section{References}

1. Chen, J. S. et al. Significance of the urokinase-type plasminogen activator and its receptor in the progression of focal segmental glomerulosclerosis in clinical and mouse models. J Biomed Sci 23 (2016).

2. Ponticelli, C. \& Glassock, R. J. Posttransplant Recurrence of Primary Glomerulonephritis. Clin J Am Soc Nephro 5, 2363-2372 (2010).

3. Jin, J., Li, Y. W. \& He, Q. Primary and Recurrent Focal Segmental Glomerulosclerosis Closely Link to Serum Soluble Urokinase-type Plasminogen Activator Receptor Levels. Transpl P 47, 1760-1765 (2015).

4. Savin, V. J. et al. Circulating factor associated with increased glomerular permeability to albumin in recurrent focal segmental glomerulosclerosis. New Engl J Med 334, 878-883 (1996).

5. Wada, T. \& Nangaku, M. A circulating permeability factor in focal segmental glomerulosclerosis: the hunt continues. Clin Kidney J 8, 708-715 (2015).

6. Deegens, J. K., Andresdottir, M. B., Croockewit, S. \& Wetzels, J. F. Plasma exchange improves graft survival in patients with recurrent focal glomerulosclerosis after renal transplant. Transpl Int 17, 151-157 (2004).

7. Zimmerman, S. W. Increased urinary protein excretion in the rat produced by serum from a patient with recurrent focal glomerular sclerosis after renal transplantation. Clin Nephrol 22, 32-38 (1984).

8. Kemper, M. J., Wolf, G. \& Muller-Wiefel, D. E. Transmission of glomerular permeability factor from a mother to her child. N Engl J Med 344, 386-387 (2001).

9. Gallon, L., Leventhal, J., Skaro, A., Kanwar, Y. \& Alvarado, A. Resolution of recurrent focal segmental glomerulosclerosis after retransplantation. N Engl J Med 366, 1648-1649 (2012).

10. Downie, M. L., Gallibois, C., Parekh, R. S. \& Noone, D. G. Nephrotic syndrome in infants and children: pathophysiology and management. Paediatr Int Child Health 37, 248-258 (2017).

11. Sharma, M. et al. Janus kinase 2/signal transducer and activator of transcription 3 inhibitors attenuate the effect of cardiotrophin-like cytokine factor 1 and human focal segmental glomerulosclerosis serum on glomerular filtration barrier. Transl Res 166, 384-398 (2015).

12. Harris, J. J. et al. Active proteases in nephrotic plasma lead to a podocin-dependent phosphorylation of VASP in podocytes via protease activated receptor-1. J Pathol 229, 660-671 (2013).

13. Delville, M. et al. A circulating antibody panel for pretransplant prediction of FSGS recurrence after kidney transplantation. Sci Transl Med 6, 256ra136 (2014).

14. Wei, C. et al. Circulating urokinase receptor as a cause of focal segmental glomerulosclerosis. Nat Med 17, 952-960 (2011).

15. Hahm, E., Peev, V. \& Reiser, J. Extrarenal determinants of kidney filter function. Cell Tissue Res 369, 211-216 (2017).

16. Zeier, M. \& Reiser, J. suPAR and chronic kidney disease-a podocyte story. Pflugers Arch 469, 1017-1020 (2017).

17. Konigshausen, E. \& Sellin, L. Circulating Permeability Factors in Primary Focal Segmental Glomerulosclerosis: A Review of Proposed Candidates. Biomed Res Int (2016).

18. Backes, Y. et al. Usefulness of suPAR as a biological marker in patients with systemic inflammation or infection: a systematic review. Intensive Care Med 38, 1418-1428 (2012).

19. Berres, M. L., Schlosser, B., Berg, T., Trautwein, C. \& Wasmuth, H. E. Soluble urokinase plasminogen activator receptor is associated with progressive liver fibrosis in hepatitis C infection. J Clin Gastroenterol 46, 334-338 (2012). 
20. Slot, O., Brunner, N., Locht, H., Oxholm, P. \& Stephens, R. W. Soluble urokinase plasminogen activator receptor in plasma of patients with inflammatory rheumatic disorders: increased concentrations in rheumatoid arthritis. Ann Rheum Dis 58, 488-492 (1999).

21. Stephens, R. W. et al. ELISA determination of soluble urokinase receptor in blood from healthy donors and cancer patients. Clin Chem 43, 1868-1876 (1997).

22. Wei, C. L. et al. Circulating suPAR in Two Cohorts of Primary FSGS. J Am Soc Nephrol 23, 2051-2059 (2012).

23. D’Agati, V. D., Fogo, A. B., Bruijn, J. A. \& Jennette, J. C. Pathologic classification of focal segmental glomerulosclerosis: A working proposal. Am J Kidney Dis 43, 368-382 (2004).

24. Amann, K. \& Haas, C. S. What you should know about the work-up of a renal biopsy. Nephrol Dial Transpl 21, 1157-1161 (2006).

25. Fogo, A. B. Causes and pathogenesis of focal segmental glomerulosclerosis. Nature Reviews Nephrology 11, 76-87 (2015).

26. Peev, V., Hahm, E. \& Reiser, J. Unwinding focal segmental glomerulosclerosis. F1000Res 6, 466 (2017).

27. Meijers, B. et al. The soluble urokinase receptor is not a clinical marker for focal segmental glomerulosclerosis. Kidney Int 85, 636-640 (2014).

28. Maas, R. J., Wetzels, J. F. \& Deegens, J. K. Serum-soluble urokinase receptor concentration in primary FSGS. Kidney Int 81, 1043-1044 (2012).

29. Sinha, A. et al. Serum-soluble urokinase receptor levels do not distinguish focal segmental glomerulosclerosis from other causes of nephrotic syndrome in children. Kidney Int 85, 649-658 (2014).

30. Maas, R. J., Wetzels, J. F. \& Deegens, J. K. Serum suPAR concentrations in patients with focal segmental glomerulosclerosis with end-stage renal disease. Kidney Int 85, 711 (2014).

31. Hayek, S. S. et al. Soluble Urokinase Plasminogen Activator Receptor and Decline in Kidney Function in Autosomal Dominant Polycystic Kidney Disease. J Am Soc Nephrol (2019).

32. Lee, J. M. et al. Increased Serum Soluble Urokinase-Type Plasminogen Activator Receptor (suPAR) Levels in FSGS: A MetaAnalysis. J Immunol Res 2019, 5679518 (2019).

33. Hayek, S. S. et al. Soluble Urokinase Receptor and Chronic Kidney Disease. New Engl J Med 373, 1916-1925 (2015).

34. Huang, J. et al. Urinary soluble urokinase receptor levels are elevated and pathogenic in patients with primary focal segmental glomerulosclerosis. BMC Med 12, 81 (2014).

35. Levey, A. S. et al. A new equation to estimate glomerular filtration rate. Ann Intern Med 150, 604-612 (2009).

36. Liu, X. Classification accuracy and cut point selection. Stat Med 31, 2676-2686 (2012).

37. Fluss, R., Faraggi, D. \& Reiser, B. Estimation of the Youden Index and its associated cutoff point. Biom J 47, 458-472 (2005).

38. Schenk, H. et al. Removal of focal segmental glomerulosclerosis (FSGS) factor suPAR using CytoSorb. JClin Apher 32, 444-452 (2017).

39. Reiser, J. \& Chapman, H. Soluble Urokinase-Type Plasminogen Activator Receptor in FSGS: Stirred but Not Shaken. Journal of the American Society of Nephrology 25, 1611-1613 (2014).

40. Spinale, J. M. et al. A reassessment of soluble urokinase-type plasminogen activator receptor in glomerular disease. Kidney Int 87, 564-574 (2015).

41. Artero, M., Biava, C., Amend, W., Tomlanovich, S. \& Vincenti, F. Recurrent Focal Glomerulosclerosis - Natural-History and Response to Therapy. American Journal of Medicine 92, 375-383 (1992).

42. Maruyama, M. et al. Focal segmental glomerular sclerosis recurrence with massive proteinuria and anuria immediately after kidney transplantation. Nephrology (Carlton) 20, 93-95 (2015).

43. Kolonko, A., Piecha, G. \& Wiecek, A. Successful Preemptive Kidney Transplantation With Rituximab Induction in a Patient With Focal Segmental Glomerulosclerosis and Massive Nephrotic Syndrome: A Case Report. Transplant Proc 48, 3092-3094 (2016).

44. Hofstra, J. M., Beck, L. H. Jr., Beck, D. M., Wetzels, J. F. \& Salant, D. J. Antiphospholipase A (2) receptor antibodies correlate with clinical status in idiopathic membranous nephropathy. Clin J Am Soc Nephrol 6, 1286-1291 (2011).

45. Palacios, C. R. F. et al. Urine But Not Serum Soluble Urokinase Receptor (suPAR) May Identify Cases of Recurrent FSGS in Kidney Transplant Candidates. Transplantation 96, 394-399 (2013).

46. Huang, J. et al. Plasma soluble urokinase receptor levels are increased but do not distinguish primary from secondary focal segmental glomerulosclerosis. Kidney Int 84, 366-372 (2013).

\section{Acknowledgements}

The study involves human participants. Plasma samples were obtained from adult study participants and/or their legal guardians older than 18 years who have given written informed consent. All methods of experiments involving human participants were carried out according to relevant guidelines and regulations and no organs/ tissues from prisoners were procured. All experimental protocols involving human participants received approval of the Ethics Committee of the Medical University of Vienna (EK 1465/2013).

\section{Author Contributions}

Study design: W.W., A.S. and G.S.P. Study conduct: W.W., G.S., H.H., C.K., A.H., Z.K., B.B., T.R., L.W., S.P., T.R., B.S. and A.S. Data collection: W.W. Data analysis: W.W. and H.H. Data interpretation: W.W. and H.H. Drafting manuscript: W.W. Revising manuscript content: W.W., G.S., H.H., C.K., A.H., Z.K., B.B., G.S.P. and A.S. Approving final version of manuscript: W.W., G.S.P., G.S., A.H., H.H., C.K., B.B., L.W., Z.K., S.P., T.R., B.S. and A.S.

Additional Information

Supplementary information accompanies this paper at https://doi.org/10.1038/s41598-019-50405-8.

Competing Interests: The authors declare no competing interests.

Publisher's note Springer Nature remains neutral with regard to jurisdictional claims in published maps and institutional affiliations.

Open Access This article is licensed under a Creative Commons Attribution 4.0 International License, which permits use, sharing, adaptation, distribution and reproduction in any medium or format, as long as you give appropriate credit to the original author(s) and the source, provide a link to the Creative Commons license, and indicate if changes were made. The images or other third party material in this article are included in the article's Creative Commons license, unless indicated otherwise in a credit line to the material. If material is not included in the article's Creative Commons license and your intended use is not permitted by statutory regulation or exceeds the permitted use, you will need to obtain permission directly from the copyright holder. To view a copy of this license, visit http://creativecommons.org/licenses/by/4.0/.

(c) The Author(s) 2019 